\title{
Time-Evolution of Polymer Spherulite Formation Investigated by Light Scattering
}

\author{
Masato HASHIMOTO ${ }^{\dagger}$ \\ Department of Polymer Science and Engineering, Faculty of Textile Science, Kyoto Institute of Technology, \\ Matsugasaki, Kyoto 606-8585, Japan
}

(Received February 16, 2004; Accepted March 17, 2004; Published August 15, 2004)

\begin{abstract}
The time-evolution of polymer spherulite formation in the melt was investigated by $H_{\mathrm{V}}$ light scattering. The $H_{\mathrm{V}}$ scattering gives both the 'four-leaf clover pattern' and isotropic scattering independent of azimuthal angle. The isotropic scattering from spherulites of isotactic polystyrene is measured over a wide range of crystallization: from bundles to a matured spherulite. Correlation length is obtained from the isotropic scattering. In the early stage of spherulite growth, the correlation length increases in proportion to crystallization time and reaches maximum value of about $1 \mu \mathrm{m}$. Hence, the isotropic scattering is attributed to the bundles. The correlation length is the size of the bundles. The bundles grow and successively evolve to spherulites by branching. In the late stage, a matured spherulite grows. However, the correlation length decreases to a value of about $0.6 \mu \mathrm{m}$ and remains unchanged. By re-examination of the lattice model of Yoon and Stein, the model is found applicable to matured spherulites. The correlation length in matured spherulites is suggested to be the distance between branches of a bundle. The length agrees to a distance between screw dislocations in a crystal grown from the polymer melt. [DOI 10.1295/polymj.36.594]

KEY WORDS Spherulite / Light Scattering / Isotropic Scattering / Isotactic Polystyrene / TimeEvolution / Correlation Length /
\end{abstract}

Light scattering is an effective tool for detecting refractive index fluctuations with small size in the length scale of $\mu \mathrm{m}$, and hence can be used in the studies of polymer crystallization. In fact, there have been many studies on internal structures of polymer spherulites $^{1-5}$ and pre-crystallization processes. ${ }^{6,7}$ Recently, Tahara et al. investigated the time evolution of poly(ethylene terephthalate) (PET) spherulite formation, ${ }^{8}$ and Hoffman and Strobl made analysis of the early stage of syndiotactic polypropylene (sPP) crystallization by using light scattering. ${ }^{9}$

A polymer spherulite usually gives the 'four-leaf clover pattern' in $H_{\mathrm{V}}$ light scattering. The pattern can be explained by the perfect spherulite model by Stein and Rhodes. ${ }^{1}$ The $H_{\mathrm{V}}$ scattering observed experimentally, however, has considerable isotropic scattering independent of azimuthal angle. The $H_{\mathrm{V}}$ scattering pattern from isotactic polystyrene (iPS) spherulites is expressed by the sum of the scattering from the perfect spherulite and the isotropic scattering. We have argued that the isotropic scattering comes from randomly oriented crystallites in $H_{\mathrm{V}}$ scattering from spherulites of iPS on the basis of the "combination model'. ${ }^{3}$ This assumes that a spherulite is comprised of the two independent components, the perfect spherulite and the randomly oriented crystallites. ${ }^{10}$

To account for the isotropic scattering, Yoon and Stein proposed a lattice model with orientational fluctuations of crystallites. ${ }^{11}$ The intensity of the isotropic scattering predicted by this model is much smaller than that observed. ${ }^{10}$

This paper reports experimental results of the isotropic scattering from isotactic polystyrene crystallized from the melt. Since iPS has a high glass transition temperature of $90^{\circ} \mathrm{C}$ and slow growth rate less than $20 \mu \mathrm{m} / \mathrm{h},{ }^{12}$ iPS can be quenched to the glass during crystallization. Hence, we can observe isolated spherulites in the glass at room temperature.

We review the lattice model of Yoon and Stein, ${ }^{11}$ re-calculate $H_{\mathrm{V}}$ scattering on the basis of the model and discuss applications of the model to spherulite growth. We finally discuss the time-evolution of polymer spherulite formation, in particular, on the basis of the crystallization time dependence of the correlation length obtained from the isotropic scattering.

\section{EXPERIMENTAL}

Sample used in this experiment was iPS purchased from Scientific Polymer Product, Inc. $\left(M_{\mathrm{w}}=5.56 \times\right.$ $\left.10^{5}, M_{\mathrm{w}} / M_{\mathrm{n}}=1.9\right)$ The iPS powder was melted between two cover-glass slides in vacuum to make films about $100 \mu \mathrm{m}$ thick. The film was melted at $270^{\circ} \mathrm{C}$ for $15 \mathrm{~min}$, self-seeded at $110^{\circ} \mathrm{C}$, crystallized isothermally at several crystallization temperatures and quenched to room temperature.

To avoid effects of truncation of spherulites by their mutual impingement, we control the number density

${ }^{\dagger}$ To whom correspondence should be addressed (E-mail: hashima@ipc.kit.ac.jp). 
of spherulite by the self-seeding method; the films of iPS is melted and cooled to $110^{\circ} \mathrm{C}$ to seed nuclei for an appropriate time, from 1 to $60 \mathrm{~min}$ and then heated to a crystallization temperature. For larger spherulites at longer crystallization time, the number density is controlled to be lower. Hence, in the light scattering experiment, the number of spherulites in the area irradiated by incident light is smaller for specimens crystallized longer. We changed specimen to specimen at one crystallization temperature according to the crystallization time; for example at a crystallization temperature of $180^{\circ} \mathrm{C}$, we used about 100 specimens. We also prepared specimens with truncated spherulites observed usually. All specimens crystallized were observed by polarized light microscopy before light scattering.

Light scattering was performed at room temperature with a light-scattering photometer (C. N. Wood Mfg. Co. Model 6000). The details of the measurement are described in a previous paper. ${ }^{10}$ In this light scattering photometer, the resolution of low scattering angle limited measurement of intensity corresponding to the four-leaf clover pattern of a spherulite with radius less than $10 \mu \mathrm{m}$. The pattern merges into a direct beam for a spherulite with larger radius.

While existence of the incubation time delays the start of crystallization, preexistence of nucleus has the finite size before crystallization. Net crystallization time for spherulites with small radius is determined by the radius of a spherulite calculated by the peak position of the four-leaf clover pattern; the crystallization time is obtained by dividing the radius by the growth rate. For longer crystallization time, crystallization time is used.

\section{RESULTS}

The intensity of the $H_{\mathrm{V}}$ light scattering from a spherulite, $I$, was expressed by the sum of intensity from the perfect spherulite, ${ }^{1} I_{\mathrm{p}}$, and the isotropic scattering, $I_{\mathrm{i}}$, independent of azimuthal angle,

$$
I=I_{\mathrm{p}}+I_{\mathrm{i}}=I_{\mathrm{p}}+\frac{A}{\left(1+k^{2} a^{2}\right)^{2}},
$$

where $A$ is a constant, $a$ is the correlation length, $k$ is the magnitude of scattering vector, $(4 \pi / \lambda) \sin (\theta / 2), \lambda$ is the wavelength in the medium and $\theta$ is the polar scattering angle. ${ }^{10}$ Since $I_{\mathrm{i}}$ is not observed from the area with no spherulites, $I_{\mathrm{i}}$ comes from spherulite itself.

Scattering pattern is found to be well fitted by a scattering curve calculated for randomly oriented crystallites with an orientational correlation length $a$ (Figure 1).

Figure 2 represents plots to obtain the correlation
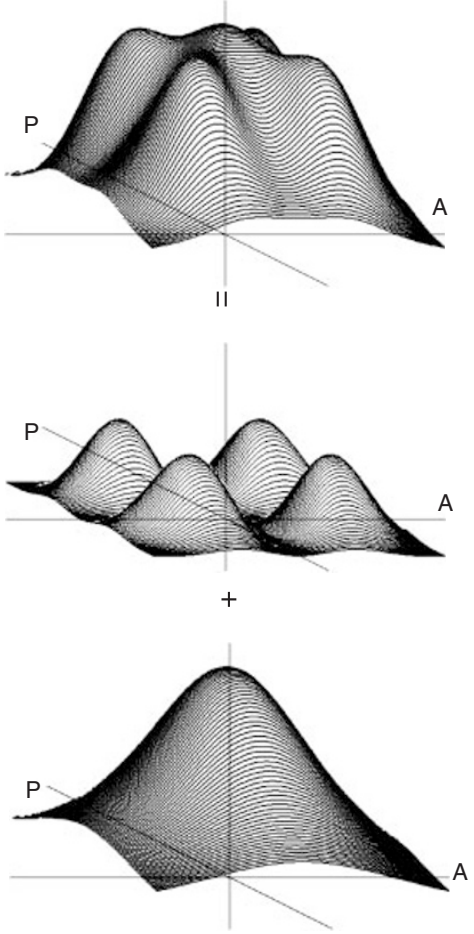

Figure 1. Bird's-eye view of $H_{\mathrm{V}}$ light scattering pattern from a spherulite; (a) experimental data, (b) scattering from a perfect spherulite, so-called 'a four-leaf clover', (c) the isotropic scattering. $\mathbf{P}$ and $\mathbf{A}$ indicate directions of the polarizer and analyzer, respectively. (a) is the sum of (b) and (c); eq 1 holds.

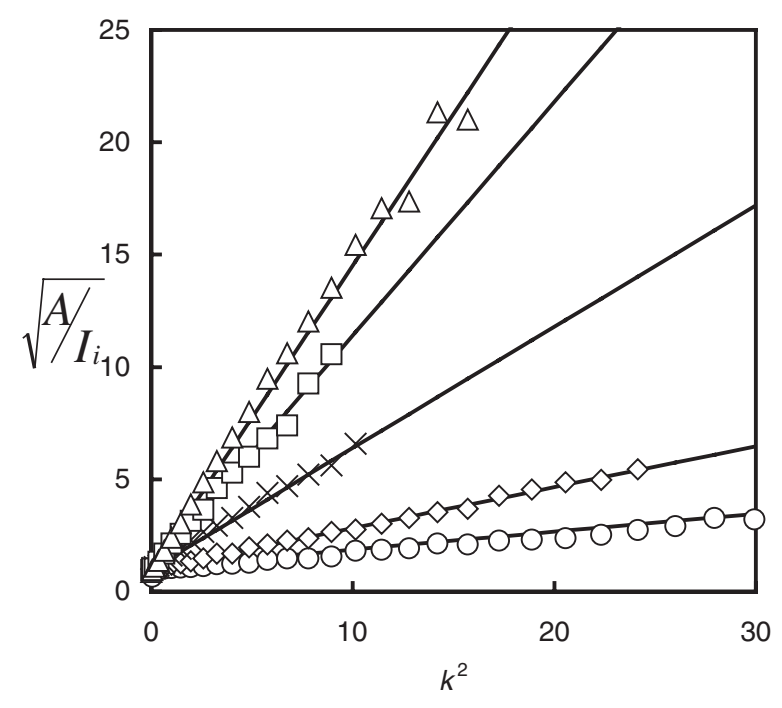

Figure 2. Plots of the square root of intensity of the isotropic scattering vs. square of $k$ for iPS spherulite grown at $180^{\circ} \mathrm{C}$ and $\bigcirc: 6 \min , \diamond: 9 \mathrm{~min}, \times: 16 \mathrm{~min}, \square: 20 \mathrm{~min}, \triangle: 24 \mathrm{~min}$.

length, $a$, utilizing the second term in eq 1 . The horizontal axis denotes the square of $k$ and vertical axis the square root of the intensity. According to eq 1, the graph should be a straight line. However, at $k=0$ the curves deviate downward from straight lines since the limit of angular resolution of photometer allows the contribution from the four-leaf clover pattern. 


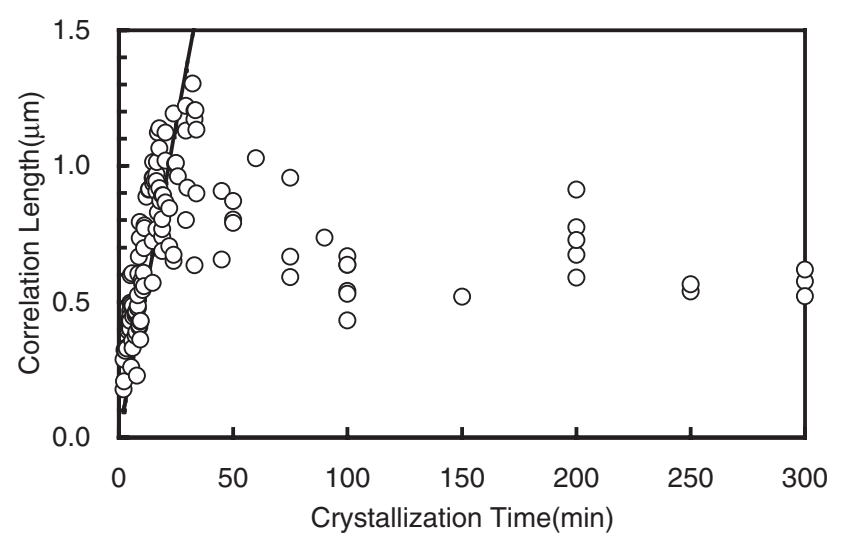

Figure 3. Plots of correlation length $v s$. crystallization time of spherulite of iPS crystallized at $180^{\circ} \mathrm{C}$. The straight line has a slope of $3 \mu \mathrm{m} / \mathrm{h}$.

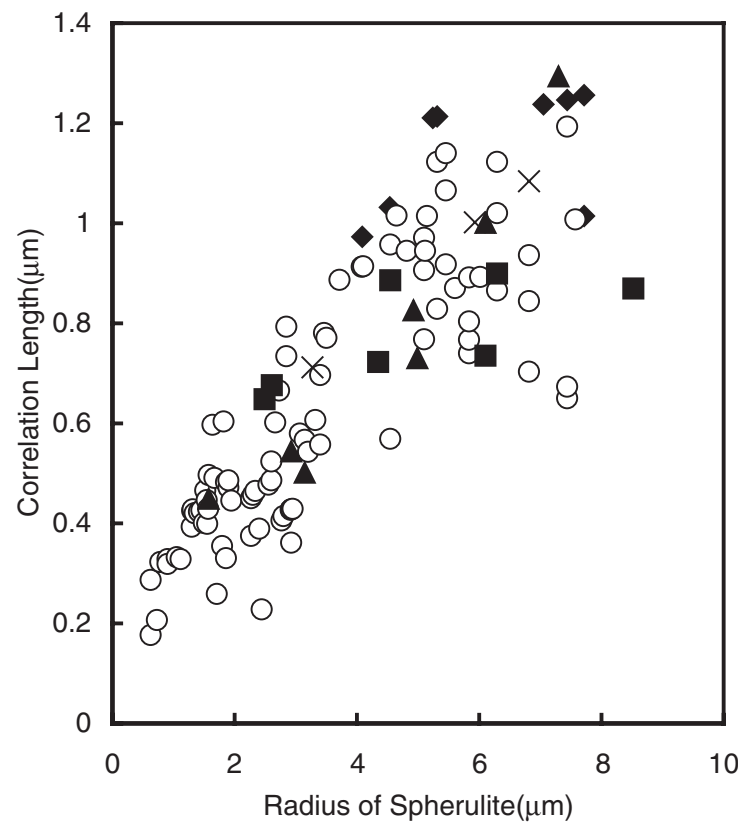

Figure 4. Plots of correlation length $v s$. radius of spherulite of iPS crystallized at $\diamond: 200^{\circ} \mathrm{C}, \bigcirc: 180^{\circ} \mathrm{C}, \boldsymbol{\square}: 160^{\circ} \mathrm{C}, \boldsymbol{\Delta}: 140^{\circ} \mathrm{C}$, $\times: 130{ }^{\circ} \mathrm{C}$.

Figure 3 shows crystallization time dependence of the correlation length for spherulites crystallized at $180^{\circ} \mathrm{C}$. Up to about $30 \mathrm{~min}$, the correlation length increases in proportion to crystallization time with a rate of about $3 \mu \mathrm{m} / \mathrm{h}$, that is, $1 / 6$ of the growth rate of an iPS spherulite. After $30 \mathrm{~min}$ the correlation length reaches maximum of about $1 \mu \mathrm{m}$. In the later stage the correlation length decreases to about $0.6 \mu \mathrm{m}$ and remains unchanged.

Figure 4 shows the dependence of the correlation length on the radius of a spherulite or axialite crystallized at $130,140,160,180$ and $200^{\circ} \mathrm{C}$. All data for different temperatures show the same dependence on the radius of spherulite.

So far we described the light scattering in the con-

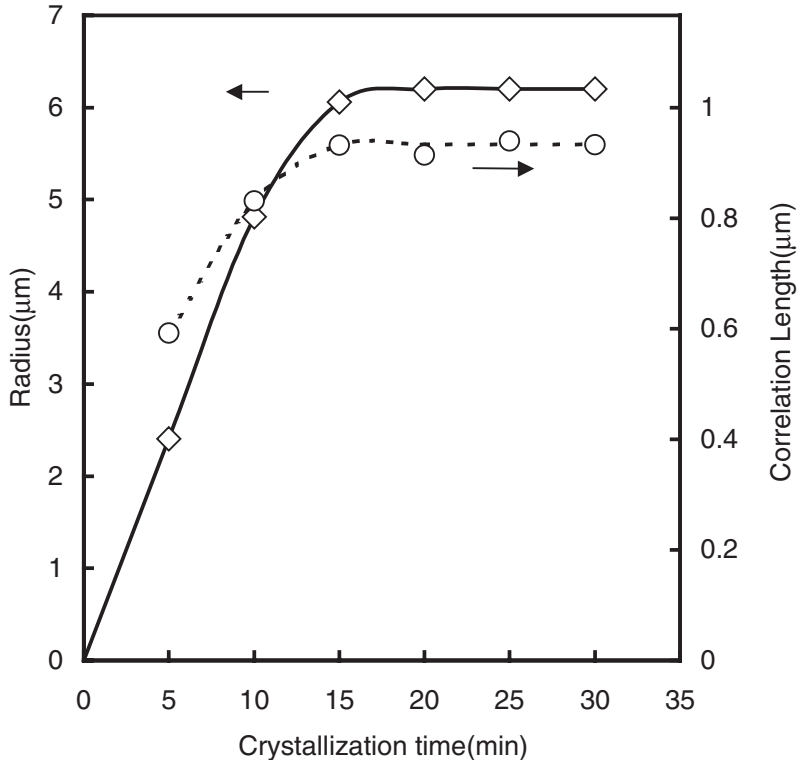

Figure 5. Plots of spherulite radius and the correlation length vs. crystallization time for truncated spherulite crystallized at $180^{\circ} \mathrm{C}(\diamond$ : radius of spherulite, $\bigcirc$ : correlation length).

dition that each spherulite is isolated in this study. Figure 5 shows crystallization time dependence of the correlation length and radius of spherulite for spherulites truncated by their mutual impingement. The optical microscopy of this specimen shows that at about $15 \mathrm{~min}$ almost all spherulites mutually impinge to be truncated; $15 \mathrm{~min}$ is much shorter than the time required for the correlation length to reach maximum in isolated spherulites. It is to be noted in Figure 5 that when the value of spherulite radius is saturated, the correlation length also ceases to increase simultaneously. The radius of spherulite thus determines the correlation length at the early stage. Annealing after the truncation has been had no effect on the correlation length.

The ratio of intensity of the isotropic scattering, $I_{\mathrm{i}}$, to the one from the perfect spherulite, $I_{\mathrm{p}}$, is an important factor of the origin of the isotropic scattering. The present experiment shows $I_{\mathrm{i}} / I_{\mathrm{p}}$, has rather a large value from 1 to 4 . The fluctuation of the ratio is attributed to statistical fluctuation due to the small number of spherulites in the irradiated area.

\section{DISCUSSION}

First, we review the two-dimensional lattice model of Yoon and Stein, since this model gives the isotropic scattering as well as the four-leaf clover pattern. The scattering intensity profile for a three-dimensional spherulite is not so much different from that for a two-dimensional one. ${ }^{11,13}$ Next, we discuss the timeevolution of polymer spherulites on the basis of the $H_{\mathrm{V}}$ isotropic scattering. 
Review of Lattice Model of Yoon and Stein ${ }^{11}$

In the model, a two-dimensional spherulite of radius $R$ is divided into a concentric lattice of fan-shaped cells, which occupy a constant area, divide the radius into $M$ cites and contain scatterers. Yoon and Stein set the following rules of placing scatterers on the lattice.

1) The first scatterer is placed in the innermost cell.

2) The cells belonging to a ring are filled in a clockwise manner.

3) Orientation of a scatterer is determined by scatterers in the nearest-neighbor cells of the same ring and of the inner ring.

4) Orientation fluctuates from the average orientation of the neighbors by an angle, $\pm \delta$ randomly. A parameter $C_{1}$ is introduced to confine the orientation around the radial direction.

Scattering amplitude from the model constructed is calculated by summing up the scattered light from all the scatterers.

It has been confirmed that in this model the correlation length is the linear dimension of a divided cell and is independent of $\delta$.

Figure 6 shows three examples of the spherulite model for $M=10, C_{1}=0.01$ and $\delta=0,0.1,0.3$. With increasing $\delta$ the 'spherulite' has more disorder structure. We performed numerical calculation of light scattering intensity for the three examples. Intensity was calculated for 100 different series of random

(a)

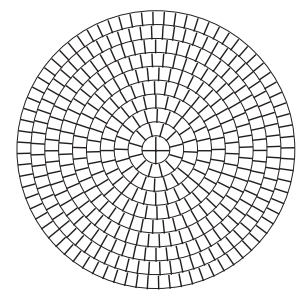

(b)

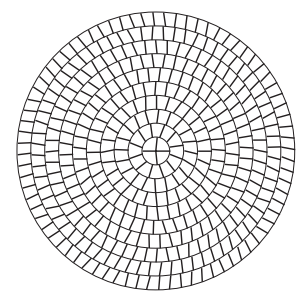

(c)

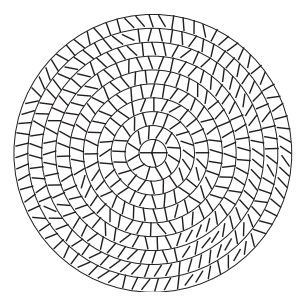

Figure 6. Three examples of the lattice model of Yoon and Stein. Thick short lines denote the principal optical axes of scatterers placed on the cells. $M=10, C_{1}=0.01$; (a) $\delta=0$, (b) $0.1,(\mathrm{c}) 0.3$. (a)

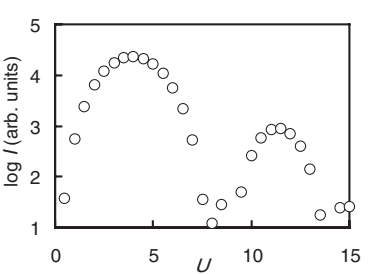

(b)

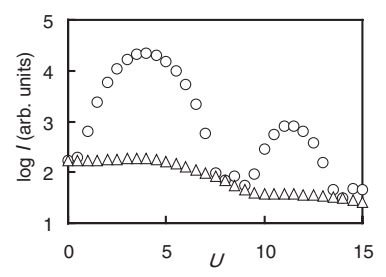

(c)

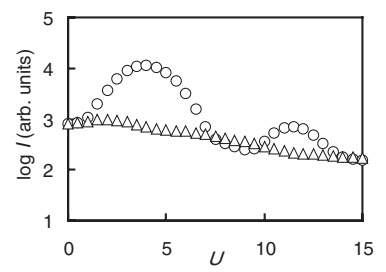

Figure 7. Numerically calculated light scattering intensity for the lattice model of Yoon and Stein. $M=10$ and $C_{1}=0.01$; (a) $\delta=0$, (b) 0.1 , (c) 0.3 . $\bigcirc$ : The intensity profile through the maximum of the four-leaf clover pattern at the azimuthal angle $\mu$ of $45^{\circ} ; \triangle$ : the isotropic scattering at $\mu=0^{\circ} . U=k R$ ( $k$ is the magnitude of scattering vector. $R$ is the radius of the spherulite).

numbers which determine probability of taking a value of fluctuation $\pm \delta$, and averaged out the results. In Figure 7 are shown the calculated results. The scattering did not agree with previous experimental results for iPS. ${ }^{10}$ Calculated ratio of the intensity of the isotropic scattering to that of the four-leaf clover pattern is much less than the ratio obtained in the early stage.

The discrepancy between calculated and experimental ratios can be explained as follows. Since $|\delta|$ is less than 1 , scattering intensity can be expanded by powers of $\delta$. The first constant term in the expansion corresponds to the intensity from the perfect spherulite. The second term proportional to $\delta$ can be averaged as zero. The isotropic scattering is the term proportional to $\delta^{2}$ and the higher powers of $\delta$. Accordingly the magnitude of the intensity of isotropic scattering is much smaller than the one from the perfect spherulite.

As will be shown later, however, the isotropic scattering calculated does correspond to that in a large spherulite in the late stage of crystallization.

\section{Early Stage of Spherulite Formation}

Time-evolution of polymer spherulite formation has early and late stages: earlier and later than $30 \mathrm{~min}$ in crystallization time at $180^{\circ} \mathrm{C}$ of crystallization temperature (Figure 3).

In the early stage till $30 \mathrm{~min}$ at $180^{\circ} \mathrm{C}$, the isotropic 
scattering is much stronger than that predicted by the lattice model of Yoon and Stein. The correlation length deduced from the isotropic scattering increases in proportion to crystallization time.

To account for strong isotropic scattering, we assume an axialitic structure in the center of spherulite. A stack of optically uni-axial crystals forms, grows with branching and increases the number of the stacked crystals. The stack of lamellar crystals was observed in iPS spherulites by electron microscopy. ${ }^{14}$ The stack of crystals gives rise to the isotropic scattering.

For a simplified model of stacked crystals, we propose an optically uni-axial sphere. In the approximation of Rayleigh and Gans, the intensity of $H_{\mathrm{V}}$ scattering from the sphere, ${ }^{15} I_{\text {sphere }}$, is given as,

$$
I_{\text {sphere }}=\frac{B}{U^{6}}(\sin U-U \cos U)^{2},
$$

where $B$ is a constant determined by the direction of the optical axis of the sphere, $U=k R(k=(4 \pi)$ $\lambda) \sin (\theta / 2)), R$ is the radius of the sphere.

It is to be noted that $B$ is independent of azimuthal angle, $\mu$. Thus $I_{\text {sphere }}$ expresses isotropic scattering. The sum of the intensity $I_{\text {sphere }}$ from many spheres oriented in arbitrary directions may show the same dependence of $U$ as that from one sphere.

For small scattering angle $(U \ll 1)$, eq 2 is approximated to the square of Lorentzian as follows,

$$
I_{\text {sphere }} \approx\left(1-\frac{U^{2}}{10}\right)^{2} \approx \frac{1}{\left(1+k^{2}\left(\frac{R}{\sqrt{10}}\right)^{2}\right)^{2}} .
$$

Here the correlation length is $R / \sqrt{10}$. However, observed correlation length is $R / 6$. Consequently, the axialitic structure in the center of a spherulite always keeps its size a half of radius of a spherulite in the early stage at all the crystallization temperatures investigated.

The optical microscopy at the early stage supports this argument. Figure 8 show polarized light micrographs of spherulites of iPS in the early stage (crystallized at $180^{\circ} \mathrm{C}$ for $15 \mathrm{~min}$ ). Rotating the specimen by every $45^{\circ}$ counterclockwise around the optical axis of a microscope, a spherulite shows a clear Maltese cross (Figure 8a), a pattern like stitch on a baseball (b), again the Maltese cross (c) and a pattern like stitch on a baseball but the direction of the stitch is rotated by $90^{\circ}$ (d). In the rotation angle giving a Maltese cross, the optical axis of the axialitic structure parallels the direction of the polarizer or analyzer. For the baseball stitch pattern, the axis of the axialitic structure makes an angle of $45^{\circ}$ with the axis of polarizer. (a)

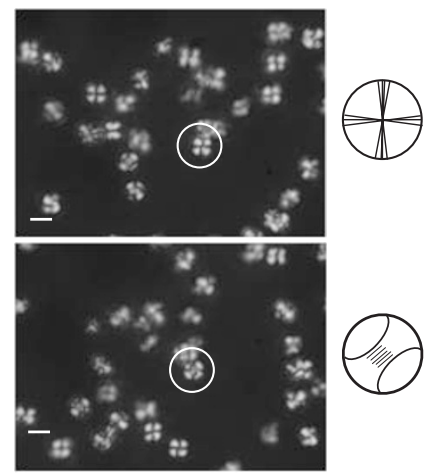

(c)

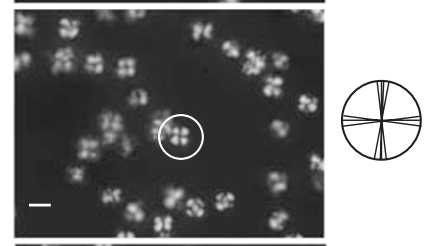

(d)

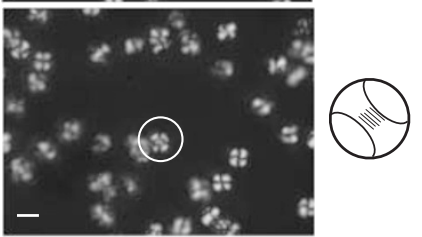

Figure 8. Polarized light micrographs of iPS spherulites crystallized at $180^{\circ} \mathrm{C}$ for $15 \mathrm{~min}$. All scale bars are $10 \mu \mathrm{m}$. Schematic illustration corresponds to a spherulite surrounded by a circle. The sample is rotated by every $45^{\circ}$ counterclockwise around the optical axis of the microscope. (a) $0^{\circ}$; (b) $45^{\circ}$; (c) $90^{\circ}$; (d) $135^{\circ}$.

Hoffman and Strobl $^{9}$ estimated the hedrite size of sPP using the Debye-Buche structure factor as the second term in eq 1 . We extend this analysis to the isotropic scattering of the axialitic structure in iPS spherulite giving a four-leaf clover pattern.

\section{Late Stage of Spherulite Formation}

At more than 30 min crystallization time, the fourleaf clover pattern shifts to lower scattering angle to merge into the direct beam. Only the isotropic scattering is observed. The origin of the isotropic scattering is different from that in the early stage. The correlation length slightly decreases and reaches a constant value of about $0.6 \mu \mathrm{m}$, while the radius of spherulite keeps growing linearly with crystallization time.

The density of the number of spherulites crystallized for longer time is lower than in the early stage. The number of the axialitic structures giving the isotropic scattering is thus small in the area irradiated by incident light. Contribution of the axialitic structure to isotropic scattering decreases with crystallization time in the present specimens to avoid the effects of the mutual impingement of spherulites. Eventually, the isotropic scattering with the correlation length of $0.6 \mu \mathrm{m}$ must be attributed not to the axialitic structure in the center but to the whole spherulite.

We propose that this isotropic scattering is just that 
calculated from the lattice model of Yoon and Stein. In the model, the divided cell size must be interpreted to be the orientational correlation length independent of the deviation angle, $\delta$, as confirmed by re-calculation with the model. The correlation length in Figure 6 is $1 / 10$ of the radius of spherulite since $M=10$.

What is the correlation length in a polymer spherulite? In a growing polymer spherulite, the branching of crystals is inevitable to fill the space. Branching must break radial symmetry to give rise to orientational disorder in the perfect spherulite structure. Hence the correlation length corresponds to the distance between branches. Branching in polymer crystals is caused by spiral growth induced by screw dislocations. The distance between screw dislocations in an iPS spherulite crystallized at $150^{\circ} \mathrm{C}$ is reported to be about $0.3 \mu \mathrm{m} .{ }^{16}$ The number density of the screw dislocation increases with decreasing crystallization temperature. Thus a branching distance in the spherulite crystallized at $180^{\circ} \mathrm{C}$ should be about $0.6 \mu \mathrm{m}$, twice that at $150{ }^{\circ} \mathrm{C}$.

\section{Time-Evolution of Polymer Spherulite Formation}

Figure 9 illustrates the time-evolution of the polymer spherulite formation in the early stage and corresponding $H_{\mathrm{V}}$ light scattering pattern. First a stack of crystals forms and develops with increasing branching to form a spherulite. The isotropic scattering is caused by the stack of crystals in the center of the spherulite,

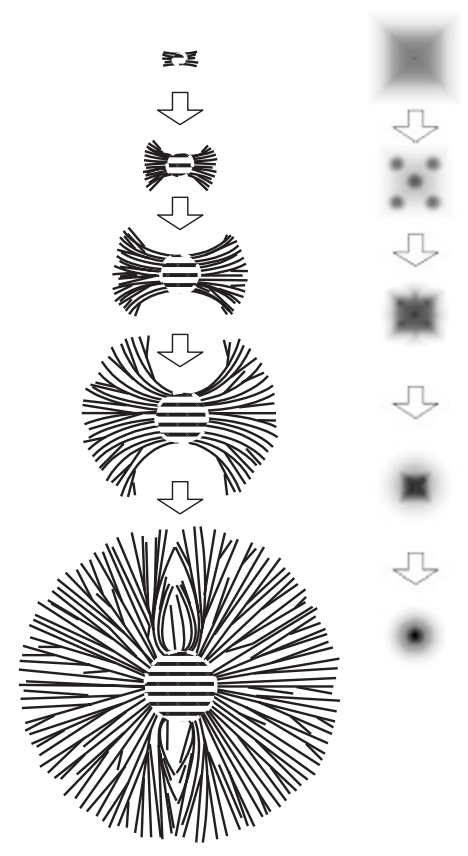

Figure 9. Schematic view of time-evolution of polymer spherulite formation and corresponding $H_{\mathrm{V}}$ light scattering patterns. the circular regions surrounded by white broken lines in the figure. The four-leaf clover pattern can be observed even for an incomplete fan-shaped spherulite ${ }^{17}$ in the early stage. The pattern shifts to lower scattering angle with crystallization time and eventually merges into a direct beam. In the late stage, only the isotropic scattering comes from orientational disorder described by the lattice model of Yoon and Stein.

Acknowledgment. The author thanks Professor $\mathrm{H}$. Miyaji of Kyoto University, Kyoto, Japan and Professor A. Toda of Hiroshima University, Hiroshima, Japan for fruitful discussion and critical readings of the manuscript. He also thanks Dr. K. Izumi of Kyoto University and Professor T. Itoh of Kyoto Institute of Technology, Kyoto, Japan for encouragement throughout this work.

\section{REFERENCES}

1. R. S. Stein and M. B. Rhodes, J. Appl. Phys., 31, 873 (1960).

2. R. S. Stein and W. Chu, J. Appl. Phys., 33, 1914 (1962).

3. A. E. M. Keijzers, J. J. van Aartsen, and W. Prins, J. Am. Chem. Soc., 90, 3167 (1968).

4. R. S. Stein and P. R. Wilson, J. Polym. Sci., Part A-2: Polym. Phys., 8, 1137 (1970).

5. T. Hashimoto, R. E. Prud'homme, and R. S. Stein, J. Polym. Sci., Polym. Phys. Ed., 11, 709 (1973).

6. G. Matsuba, K. Kaji, K. Nishida, T. Kanaya, and M. Imai, Polym. J., 31, 722 (1999).

7. M. Imai, K. Kaji, T. Kanaya, and Y. Sakai, Phys. Rev. B, 52, 12696 (1995).

8. D. Tahara, K. Fukao, and Y. Miyamoto, Polymer, 43, 7461 (2002).

9. A. Hoffmann and G. Strobl, Polymer, 44, 5809 (2003).

10. M. Hashimoto, A. Toda, and H. Miyaji, Polymer, 33, 909 (1992).

11. D. Y. Yoon and R. S. Stein, J. Polym. Sci., Polym. Phys. Ed., 36, 763 (1965).

12. T. Suzuki and A. J. Kovacs, Polym. J., 1, 82 (1970).

13. M. Matsuo and K. Ihara, J. Polym. Sci., Polym. Phys. Ed., 20, 1 (1982).

14. A. S. Vaughan and D. C. Bassett, "Morphology of Polymers," in Proceedings 17th Europhysics Conference on Macromolecular Physics, Prague, Czechoslovakia, July 15-18, 1985, B. Sedlacek, Ed., Walter de Gruyter, Inc., Berlin, 1986.

15. H. C. van de Hulst, "Light Scattering by Small Particles," Dover Publications, Inc., New York, N.Y., 1957, chap 7.

16. K. Izumi, Gan Ping, M. Hashimoto, A. Toda, H. Miyaji, Y. Miyamoto, and Y. Nakagawa, in "Advances in the Understanding of Crystal Growth Mechanics," T. Nishinaga, K. Nishioka, J. Harada, A. Sasaki, and H. Takei, Ed., Elsevier Science, New York, N.Y., 1997, p 337.

17. C. Picot, R. S. Stein, M. Motegi, and H. Kawai, J. Polym. Sci., Part A-2: Polym. Phys., 8, 2115 (1970). 